\title{
A GENERALIZATION OF THE STONE-WEIERSTRASS THEOREM
}

\section{ERRETT BishoP}

1. Introduction. Consider a compact Hausdorff space $X$ and the set $C(X)$ of all continuous complex-valued functions on $X$. Consider also a subset $\mathfrak{A}$ of $C(X)$ which is an algebra, which is closed in the uniform topology of $C(X)$, which contains the constant functions, and which contains sufficiently many functions to distinguish points of $X$. Such an algebra $\mathfrak{A}$ is called self-adjoint if the complex conjugate of each function in $\mathfrak{A}$ is in $\mathfrak{A}$. The classical Stone-Weierstrass Theorem states that if $\mathfrak{A}$ is self-adjoint then $\mathfrak{U}=C(X)$. If $\mathfrak{A}$ has the property that the only functions in $\mathfrak{A}$ which are real at every point of $X$ are the constant functions then $\mathfrak{A}$ is called anti-symmetric. Clearly antisymmetry and self-adjointness are opposite properties, in the sense that if $\mathfrak{A}$ has both properties then $X$ must consist of a single point.

Hoffman and Singer [2] have studied these two properties and given several interesting examples. The present paper was inspired by their work but it more directly relates to a previous paper of Šilov [3]. The purpose of the present paper is to prove the following decomposition theorem for a general algebra $\mathfrak{A}$ of the type defined above.

THEOREM. There exists a partition $P$ of $X$ into disjoint closed sets such that

(i) for each $S$ in $P$ the restriction $\mathfrak{U}_{S}$ of $\mathfrak{U}$ to $S$ is anti-symmetric,

(ii) if a function $f$ in $C(X)$ has, for each $S$ in $P$, a restriction to $S$ which belongs to $\mathfrak{U}_{S}$, then $f$ is in $\mathfrak{A}$,

(iii) for each $S$ in $P$, each closed subset $T$ of $X-S$, and each $\varepsilon>0$ there exists $g$ in $\mathfrak{U}$ with $\|g\| \leqq 1$, with $|g(x)-1|<\varepsilon$ for $x$ in $S$, and with $|g(x)|<\varepsilon$ for $x$ in $T$.

Property (ii) of this theorem is the essential new fact of this paper. The construction given below which leads to the partition $P$ is due to Šilov [3], who in essence proved (i) and (iii). Šilov proved a weaker property than (ii). Our proofs are different from those of Silov, although the construction is the same.

The fact that the Stone-Weierstrass theorem is a special case of the theorem to be proved here is clear. If $\mathfrak{U}$ is self-adjoint then each $\mathfrak{U}_{S}$ is self-adjoint. Since $\mathfrak{A}_{S}$ is also anti-symmetric, each set $S$ in $P$ consists of a single point. Therefore $\mathfrak{A}_{S}=C(S)$. By the theorem to

Received June 15, 1960. 
be proved, it follows that each function in $C(X)$ is in $\mathfrak{A}$. Thus $\mathfrak{A}=C(X)$, which is the conclusion of the Stone-Weierstrass Theorem.

2. Proof of the theorem. The key step in the proof will be the following lemma.

Lemma. Let $Y$ be a compact Hausdorff space and $\mathfrak{B}$ be a subalgebra of $C(Y)$ which contains the constant functions. Let $\mathfrak{R}$ be all real functions in $\mathfrak{B}$. Define $y_{1} \equiv y_{2}$, for $y_{1}$ and $y_{2}$ in $Y$, to mean that $f\left(y_{1}\right)=f\left(y_{2}\right)$ for all $f$ in $\Re$. Let $Q$ be the set of all equivalence classes for this equivalence relation, so that $Q$ is a partition of $Y$ into disjoint closed sets. Let $\mu$ be a finite complex-valued Baire measure on $Y$ and $f$ a function in $C(Y)$ such that

(a) $\|\mu\| \leqq 1$,

(b) $\int g d \mu=0$ for all $g$ in $\mathfrak{B}$,

(c) $\quad \int f d \mu \neq 0$.

Then there exists $S_{0}$ in $Q$ and a finite complex-valued Baire measure $\nu$ on $S_{0}$ such that
$\left(a_{1}\right) \quad\|\nu\| \leqq 1$,
$\left(b_{1}\right) \quad \int g d \nu=0$ for all $g$ in $\mathfrak{B}$,
(c) $\quad\left|\int f d \nu\right| \geqq\left|\int f d \mu\right|$.

Proof. It is clearly no loss of generality to assume that $\mathfrak{B}$ is closed in $C(Y)$. Let $\gamma=\left\{g_{i}\right\}$ be a finite set of functions in $\Re$ such that

$$
\begin{gathered}
g_{i} \geqq 0 \text { for all } i, \\
\Sigma g_{i}=1 .
\end{gathered}
$$

Let $\Gamma$ denote the class of all such $\gamma$. Define a partial ordering on $\Gamma$ by writing

$$
\left\{g_{i}\right\} \leqq\left\{g_{j}^{\prime}\right\}
$$

if there exists a mapping $\varphi$ of the set of indices $j$ onto the set of indices $i$ such that

$$
g_{i}=\sum_{\varphi(j)=i} g_{j}^{\prime}
$$

for all $i$. To see that $\Gamma$ is a directed set relative to this partial ordering, let $\left\{g_{i}\right\}$ and $\left\{g_{j}^{\prime}\right\}$ be any two elements of $\Gamma$. Then the set $\left\{g_{i} g_{j}^{\prime}\right\}$ is clearly a common successor of $\left\{g_{i}\right\}$ and $\left\{g_{j}^{\prime}\right\}$.

Consider $\gamma=\left\{g_{i}\right\}$ in $\Gamma$. For each index $i$ let $\mu_{i}$ be the measure 
defined by

$$
\mu_{i}(H)=\int_{H} g_{i} d \mu
$$

for each Baire subset $H$ of $S$. Clearly

$$
\Sigma\left\|\mu_{i}\right\|=\|\mu\| \leqq 1
$$

and

$$
\int f d \mu=\sum \int f d \mu_{i}
$$

Thus for at least one value of $i$ with $\left\|\mu_{i}\right\| \neq 0$ we have

$$
\left|\int f d \mu_{i}\right| /\left\|\mu_{i}\right\| \geqq\left|\int f d \mu\right| \text {. }
$$

Choose such a value of $i$ and write

$$
\mu_{\gamma}=\frac{\mu_{i}}{\left\|\mu_{i}\right\|}
$$

It follows that

$$
\left\|\mu_{\gamma}\right\|=1
$$

and

$$
\left|\int f d \mu_{\gamma}\right| \geqq\left|\int f d \mu\right|
$$

By the compactness in the weak* topology of the unit sphere of the set of Baire measures on $X$, it follows that the net $\left\{\mu_{\gamma}\right\}$ has a cluster point $\nu$ in the weak* topology. Let $x_{0}$ be any point in the support of the measure $\nu$, and let $S_{0}$ be that member of the partition $P$ which contains $x_{0}$. Let $x_{1}$ be any point in $X-S_{0}$. Thus there exists $h_{0}$ in $\Re$ with $h_{0}\left(x_{0}\right) \neq h_{0}\left(x_{1}\right)$. Let

$$
h_{1}=c_{1} h_{0}+c_{2}
$$

If the real constants $c_{1}$ and $c_{2}$ are chosen properly then

$$
h_{1}\left(x_{1}\right)=1, \quad h_{1}\left(x_{0}\right)=0 .
$$

It follows that there exists a neighborhood $U_{0}$ of $x_{0}$ and a neighborhood $U_{1}$ of $x_{1}$ such that

$$
h_{1}(x)<\frac{1}{4}, \quad x \in U_{0}
$$

and 


$$
h_{1}(x)>\frac{3}{4}, \quad x \in U_{1}
$$

Let $\lambda$ be continuous real-valued function on the range of $h_{1}$ with $0 \leqq$ $\lambda \leqq 1, \lambda(t)=0$ for $t \leqq \frac{1}{4}, \lambda(t)=1$ for $t \geqq \frac{1}{4}$. By the Weierstrass approximation theorem, $\lambda(t)$ is a uniform limit of polynomials in $t$. Therefore the function

$$
h_{2}=\lambda \cdot h_{1}
$$

is in $\Re$. Clearly $0 \leqq h_{2} \leqq 1, h_{2}(x)=0$ for $x$ in $U_{0}$, and $h_{2}(x)=1$ for $x$ in $U_{1}$.

Define $g_{1}=h_{2}$ and $g_{2}=1-h_{2}$, so that $\left\{g_{i}\right\} \in \Gamma$. If $\gamma=\left\{g_{j}^{\prime}\right\}$ is an element of $\Gamma$ which follows $\left\{g_{i}\right\}$, then each $g_{j}^{\prime}$ vanishes on either $U_{0}$ or $U_{1}$. Therefore the support of $\mu_{\gamma}$ is either a subset of $X-U_{0}$ or of $X-U_{1}$. Thus the support of $\nu$ is either a subset of $X-U_{0}$ or $X-U_{1}$. By the choice of $x_{0}$, it follows that the support of $\nu$ cannot be a subset of $X-U_{0}$ and is therefore a subset of $X-U_{1}$. Therefore $x_{1}$ is not in the support of $\nu$. Since $x_{1}$ was any point in $X-S_{0}$, it follows that the support of $\nu$ is a subset of $S_{0}$. Thus $\nu$ is a Baire measure on $S_{0}$. It is clear from the definition of $\nu$ and from $(\alpha)$ and $(\beta)$ that $\left(a_{1}\right)$ and $\left(c_{1}\right)$ are valid. It only remains to prove $\left(b_{1}\right)$. Now for each $g$ in $\mathfrak{B}$ and each $\gamma$ in $\Gamma$ we have

$$
\int g d \mu_{\gamma}=\int g g_{i} d \mu=0
$$

by (b) and the fact that $g g_{i} \in \mathfrak{B}$. Passing to the limit gives $\left(b_{1}\right)$. This completes the proof of the lemma.

Let $\Omega$ be the class of ordinal numbers whose cardinal numbers are less than or equal to $2^{\beta}$, where $\beta$ is the cardinal number of $X$. For each $\sigma$ in $\Omega$ we define by transfinite induction a partition $P_{\sigma}$ of $\Omega$ into. disjoint closed sets. This is to be done in such a way that $P_{\sigma}$ is a refinement of $P_{\tau}$ for $\sigma>\tau$. The definition is started by defining $P_{1}=\{X\}$, so that the first partition $P_{1}$ consists of the set $X$ alone. Assume that $P_{\tau}$ has been defined for all ordinals $\tau<\sigma$. If $\sigma$ has a predecessor $\sigma_{0}$, let $S$ be any element of $P_{\sigma_{0}}$, and let $\mathfrak{F}$ be the set of all functions in I which are real on $S$. Partition $S$ by defining $x_{1} \equiv x_{2}$ for $x_{1}$ and $x_{2}$ in $S$ to mean that $f\left(x_{1}\right)=f\left(x_{2}\right)$ for all $f$ in $\mathfrak{F}$. Clearly $S$ is partitioned into disjoint closed sets by this equivalence relation. The totality of all sets into which the elements $S$ of $P_{\sigma_{0}}$ are partitioned in this way is defined to be the class $P_{\sigma}$.

If $\sigma$ has no predecessor, define $x_{1} \equiv x_{2}$, for $x_{1}$ and $x_{2}$ in $X$, to mean that $x_{1}$ and $x_{2}$ belong to the same element of $P_{\tau}$ for all $\tau<\sigma$. The equivalence classes of this equivalence relation clearly form a partition $P_{\sigma}$ of $X$ into disjoint closed sets. Thus the classes $P_{\sigma}$ are defined for 
all $\sigma$ in $\Omega$, and it is clear that $P_{\sigma}$ is a refinement of $P_{\tau}$ whenever $\sigma>\tau$.

Assume that $P_{\sigma+1}$ is a proper refinement of $P_{\sigma}$ for all $\sigma$ in $\Omega$, i.e., that $P_{\sigma+1} \neq P_{\sigma}$. Then $P_{\sigma+1}$ contains a set not in any $P_{\tau}$ for $\tau<\sigma+1$. Therefore the cardinal number of subsets of $X$ is at least equal to the cardinal number of the set $\Omega$. This contradicts the choice of $\Omega$. Therefore there exists an ordinal in $\rho$ such that $P_{\rho+1}=P_{\rho}$. We shall show that the partition $P=P_{\mathrm{p}}$ satisfies all requirements of the theorem.

The fact (i) that $\mathfrak{A}_{S}$ is anti-symmetric for each $S$ in $P=P_{\rho}$ is a consequence of the fact $P_{\rho}=P_{\rho+1}$.

We next prove (ii). Consider to this end $f$ in $C(X)$ such that the restriction of $f$ to $S$ belongs to $\mathfrak{A}_{S}$ for all $S$ in $P$. Assume that $f$ is not in $\mathfrak{A}$. By the Hahn-Banach theorem, there exists a bounded linear functional on $C(X)$ which vanishes on $\mathfrak{U}$ and does not vanish at $f$. By the Riesz representation theorem, this functional can be realized as a measure $\mu$ on $X$. Thus

$$
\begin{aligned}
& \int g d \mu=0, \quad g \in \mathfrak{A} \\
& \int f d \mu \neq 0 .
\end{aligned}
$$

we may clearly assume that $\|\mu\| \leqq 1$. We now construct, by transfinite induction, a set $S_{\sigma}$ in $P_{\sigma}$ for each $\sigma$ in $\Omega$ and a finite complex-valued Baire measure $\mu_{\sigma}$ on $S_{\sigma}$ with

(1) $S_{\sigma} \subset S_{\tau}$ for $\tau<\sigma$,

(2) $\left|\int f d \mu_{\sigma}\right| \geqq\left|\int f d \mu\right|$,

(3) $\left\|\mu_{\sigma}\right\| \leqq 1$,

(4) $\int g d \mu_{\sigma}=0$, all $g$ in $\mathfrak{x}$.

Clearly the measure $\mu_{1}=\mu$ does the trick for $\sigma=1$. Assume therefore that the sets $S_{\tau}$ and the measures $\mu_{\tau}$ have been constructed for all $\tau<\sigma$. If $\sigma$ is a limit ordinal, let

$$
S_{\sigma}=\bigcap_{\tau<\sigma} S_{\tau}
$$

and let $\mu_{\sigma}$ be any cluster point in the weak* topology of the net $\left\{\mu_{\tau}\right\}_{\tau<\sigma}$. Clearly this set $S_{\sigma}$ and the measure $\mu_{\sigma}$ do the trick.

If $\sigma$ is not a limit ordinal, then there exists $\tau$ with $\sigma=\tau+1$. Let $Y$ be $S_{\tau}$ and let $\mathfrak{B}$ be the restriction to $Y$ of the algebra $\mathfrak{A}$. By the above lemma, applied to the measure $\mu_{\tau}$, there exists a finite complexvalued Baire measure $\mu_{\sigma}$ on some $S_{\sigma} \in P_{\sigma}$, with $S_{\tau} \subset Y=S_{\sigma}$, such that

(3) $\left\|\mu_{\sigma}\right\| \leqq 1$,

(4) $\int g d \mu_{\sigma}=0$, all $g$ in $\mathfrak{B}$ 
( 2) $\left|\int f d \mu_{\sigma}\right| \geqq\left|\int f d \mu_{\tau}\right| \geqq|f d \mu|$.

Thus the set $S_{\sigma}$ and the measure $\mu_{\sigma}$ do the trick.

This completes the construction of the sets $S_{\sigma}$ and the measures $\mu_{\sigma}$. For $\sigma=\rho$ we have

$$
\left|\int f d \mu_{\rho}\right| \geqq\left|\int f d \mu\right| \neq 0
$$

and $\int g d \mu_{\rho}=0$ for all $g$ in $\mathfrak{A}$. Therefore the restriction of $f$ to $S_{\rho}$ is not in $\mathfrak{A}_{S_{\rho}}$. This contradicts the assumption of (ii), thereby proving that $f$ is in 2 . This proves (ii).

It remains to prove (iii). To do this we prove by induction on $\sigma$ that for each $\sigma$ in $\Omega$, each $S$ in $P_{\sigma}$, each closed subset $T$ of $X-S$, and each $\varepsilon>0$ there exists $g$ in $\mathfrak{U}$ with $\|g\| \leqq 1,|g(x)-1|<\varepsilon$ for all $x$ in $S$, and $|g(x)|<\varepsilon$ for all $x$ in $T$. Once this is done (iii) is obviously obtained by letting $\sigma$ equal $\rho$. Since the existence of $g$ is clear if $\sigma=1$, consider $\sigma>1$ and assume that all smaller values of $\sigma$ have been disposed of. If $\sigma$ is a limit ordinal, there exists $\tau<\sigma$ and $R$ in $P_{\tau}$ with $S \subset R$ and $T \subset X-R$. By the induction hypothesis, there exists $g$ in $\mathfrak{A}$ with $\|g\| \leqq 1,|g(x)-1|<\varepsilon$ for all $x$ in $R$, and $|g(x)|<\varepsilon$ for all $x$ in $T$. Since $S \subset R$ this function $g$ has the required properties.

It remains to consider the case of an ordinal $\sigma$ which has a predecessor $\tau$, so that $\sigma=\tau+1$. Let $R$ be that element of $P_{\tau}$ for which $S \subset R$. Let (D) be the closure in $C(R)$ of $\mathfrak{U}_{R}$. Let $x_{1}$ be any point in $S$ and $x_{0}$ any point in $T \cap R$. By the definition of the partition $P_{\sigma}$ there exists $h_{1}$ in $\mathfrak{U}$ such that the restriction of $h_{1}$ to $\Re$ is real and such that $h_{1}\left(x_{0}\right) \neq h_{1}\left(x_{1}\right)$. Let $\lambda$ be a function on $h_{1}(R)$ with $0 \leqq \lambda \leqq 1, \lambda\left(h_{1}\left(x_{0}\right)\right)=0, \lambda\left(h_{1}\left(x_{1}\right)\right)=1$. Define the function $h_{2}$ in $C(R)$ by

$$
h_{2}=\lambda \circ h_{1} \text {. }
$$

Since $\lambda$ is a uniform limit of polynomials, $h_{2} \in \mathfrak{D}$. Clearly $\left\|h_{2}\right\| \leqq 1$, $h_{2}\left(x_{0}\right)=0$, and $h_{2}\left(x_{1}\right)=1$. Thus $h_{2}(x)=1$ for all $x$ in $S$. By the compactness of $T \cap R$, the product of a certain finite number of such functions $h_{2}$ will be a function $h_{3}$ in $\mathfrak{D}$ with $\left\|h_{3}\right\| \leqq 1, h_{3}(x)=1$ for $x$ in $S$, $\left|h_{3}(x)\right|<\delta$ for $x$ in $T \cap R$, where $\delta$ is an arbitrarily small positive number. By the definition of $\mathfrak{D}$, there exists $h_{4}$ in $\mathfrak{A}$ with

$$
\left|h_{3}(x)-h_{4}(x)\right|<\delta
$$

for all $x$ in $R$. Define

$$
h_{5}=(1+2 \delta)^{-1} h_{4},
$$

so that $h_{5} \in \mathfrak{X}$. Also

$$
\left|h_{5}(x)\right| \leqq(1+2 \delta)^{-1}\left(\left|h_{3}(x)\right|+\delta\right)<1
$$


for all $x$ in $R$. Thus there exists an open set $U_{0} \subset X$ with $R \subset U_{0}$ such that $\left|h_{5}(x)\right|<1$ for all $x$ in $U_{0}$. If $\delta$ is sufficiently small it is clear that

$$
\left|h_{5}(x)-1\right|<\frac{\varepsilon}{2}
$$

for all $x$ in $S$ and

$$
\left|h_{5}(x)\right|<\varepsilon
$$

for all $x$ in $T \cap R$. Thus there exists an open set $U_{1}$ in $X$ with $T \cap R \subset U_{1}$ such that $\left|h_{5}(x)\right|<\varepsilon$ for all $x$ in $U_{1}$. By the induction hypothesis at stage $\tau$ there exists $h_{6}$ in $\mathfrak{A}$ with $\left\|h_{6}\right\| \leqq 1,\left|h_{6}(x)-1\right|<$ $\varepsilon / 2$ for $x$ in $R$,

$$
\left|h_{6}(x)\right|<\min \left\{\left|h_{5}(x)\right|^{-1}: x \in X-U_{0}\right\}
$$

for all $x$ in $X-U_{0}$, and

$$
\left|h_{6}(x)\right|<\min \left\{\varepsilon\left|h_{5}(x)\right|^{-1}: x \in T-U_{1}\right\},
$$

for all $x$ in $T-U_{1}$.

Define $g=h_{5} h_{6}$, so that $g \in \mathfrak{A}$. For $x$ in $U_{0}$ we have $\left|h_{5}(x)\right| \leqq 1$, so that $|g(x)| \leqq 1$ since $\left\|h_{6}\right\| \leqq 1$. For $x$ in $X-U_{0}$ we have $|g(x)| \leqq 1$ because of $(*)$. Thus $\|g\| \leqq 1$. For $x$ in $S$ we have $\left|h_{6}(x)-1\right|<\varepsilon / 2$ and $\left|h_{5}(x)-1\right|<\varepsilon / 2$, so that

$$
|g(x)-1| \leqq\left|h_{6}(x)\right|\left|h_{5}(x)-1\right|+\left|h_{6}(x)-1\right|<\varepsilon .
$$

For $x$ in $U_{1}$ we have $\left|h_{5}(x)\right|<\varepsilon$, so that $|g(x)|<\varepsilon$ since $\left\|h_{6}\right\| \leqq 1$. For $x$ in $T-U_{1}$ we have $(\stackrel{*}{*})$, so that $|g(x)|<\varepsilon$. Thus $|g(x)|<\varepsilon$ for all $x$ in $T$. Thus $g$ has all of the required properties. This completes the proof of (iii) and thereby the proof of the theorem.

We note in conclusion that property (iii) and some results to be found in [1] imply that $\mathfrak{A}_{S}$ is closed in $C(S)$ for each $S$ in $P$.

\section{REFERENCES}

1. H. S. Bear, Some boundary properties of function algebras, Proc. Amer. Math. Soc., 11 (1960), 1-4.

2. K. Hoffman and I. Singer, On some conjectures of Gelfand (in Russian), Uspehi Math. Nauk (N.S.), 14 (1959), 99-114.

3. G. E. Šilov, On rings of functions with uniform convergence (in Russian), Ukrainian Math. Journal, 3 (1951), 404-411. 
